\title{
Evaluation of effective stress along the border of lateral notches
}

\author{
E. MAGGIOLINI, R. TOVO and P. LIVIERI \\ Department of Engineering, University of Ferrara, via Saragat 1, 44122 Ferrara, Italy
}

Received Date: 1 February 2016; Accepted Date: 5 March 2016; Published Online: 2016

\begin{abstract}
A B S TRACT This paper analyses the issue of defect nucleation due to fatigue loading in notched components where cracks occur in the proximity of the zone where the major stress gradient occurs. On the basis of recent experimental results where the defects do not nucleate at the notch tip, the effective stress evaluated by means of the implicit gradient approach, can explain the reasons for this apparent discrepancy.

Furthermore, in order to find a kind of notch that underlines the nucleation outside the maximum stress zone, rectangular lateral notches have been investigated. Rectangular notches could provide an interesting theoretical and experimental benchmark or reference case in order to validate effective stress definitions. The linear elastic stress field at edges, corners and in the surrounding material of rectangular, sharp or rounded lateral notches has been analysed by means of the implicit gradient approach. The consequent effective values of these notches are evaluated in relation to brittle fractures or their predicted fatigue strength values.
\end{abstract}

Keywords stress analysis, notches; equivalent stress; local field; implicit gradient.

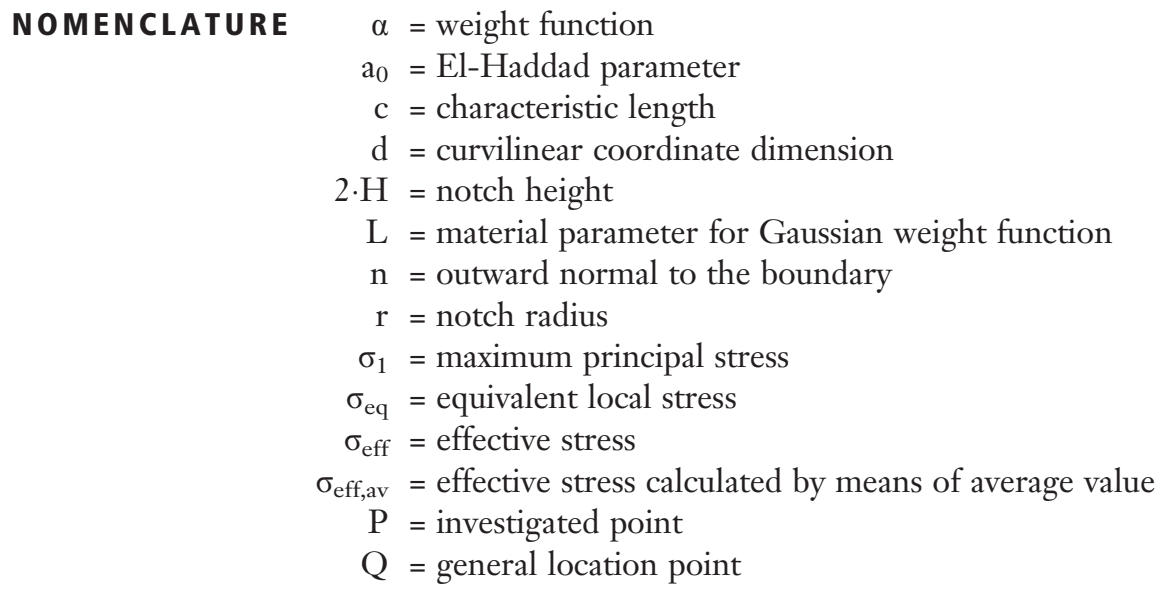

\section{INTRODUCTION}

The traditional linear elastic fracture mechanics consider the propagation of defects and evaluate crack growth from the crack tip. The crack tip is always considered as the critical point because the stress components become singu$\operatorname{lar}^{1,2}$ and the loading can only change the direction of the incremental crack at the tip. ${ }^{3,4}$ For rounded notches Neuber $^{5}$ and Peterson ${ }^{6}$ are highly renowned in the field, because, more than 50 years ago, they provided the first

Correspondence: P. Livieri, E-mail: Paolo.livieri@unife.it solutions (in relation to just a few simple cases) for Unotches of different shapes. Since then, a great deal of research focused on these kinds of notches, characterising them according to the depth and radius of the notch. ${ }^{7,8}$

In practice, shoulder fillets (a shaft is a typical example) and U-notches (for example in relation to keyways) are commonly used and much research is being carried out worldwide to understand the mechanisms underlying the irregularity of the geometry and the material behaviour in their near fields. Shoulder fillets are more frequently used than U-notches; especially in high performance structures, where designers tend to introduce sudden reductions in 


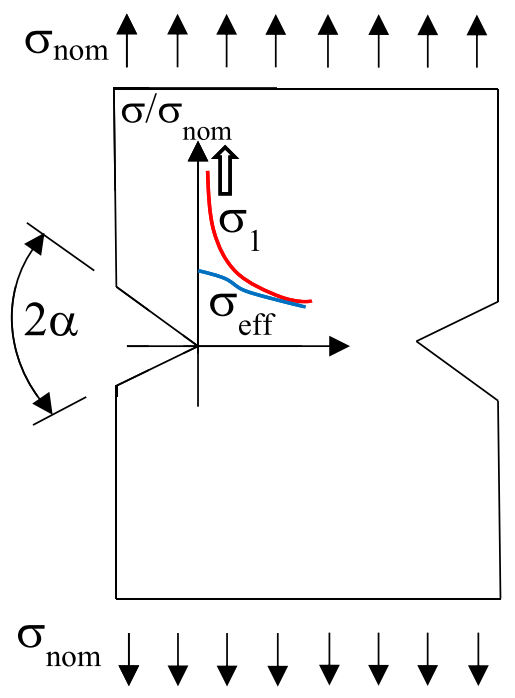

Fig. 1 Stress field along the bisector. Principal stress $\sigma_{1}$, and effective stress $\sigma_{\text {eff. }}$

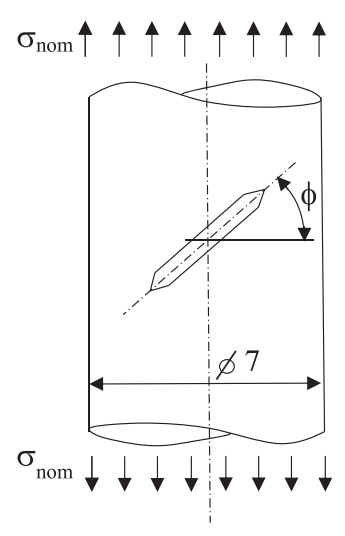
$\phi=0^{\circ}$

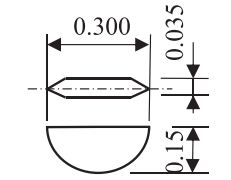

$\phi=30^{\circ}$
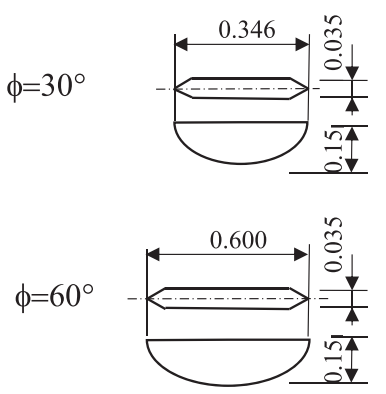

Fig. 2 Shapes and dimensions of defects in a cylindrical body under tensile loading experimental analysed by Lorenzino et al. ${ }^{9}$ (dimension in millilitres)

section and thickness. A well-known problem associated with the use of notches regards their stress concentration factors. In the worst case scenario, notches can even result in a stress singularity with all its associated problems.

In a recent paper, Lorenzino et al. ${ }^{9}$ considered the problem of small tilted sharp defects under fatigue loadings. They observed that, for tilted defects, the cracks did not initiate at the notch tip but at a certain distance from the tip, whereas, when the crack was perpendicular to the load direction, the nucleation zone agrees exactly at the notch tip. A similar behaviour was observed in the scientific literature from researchers who studied the fatigue behaviour of spot welded joints under fatigue loadings. ${ }^{10}$ The cracks can move to the outside of the heataffected zone in medium- and low-cycle fatigue. ${ }^{11,12}$ This phenomena was just underlined in reference ${ }^{13}$ and analysed by means of the implicit gradient approach.

Over the years, the precision of mechanical tools has increased dramatically, resulting in very small notch radii that often give a singular stress field. ${ }^{14}$ When the stress concentration factors tends towards infinite the maximum local stress can not used for fatigue prediction. For this reason, energy based criteria (strain energy densities ${ }^{15,16}$ ) average stress field approaches (implicit gradient $^{17,18}$ and critical distance theory ${ }^{19,20}$ ) are becoming more important. U-notches of various shapes and under different load directions have been studied over recent years, ${ }^{21,22}$ nevertheless the Brazilian disk has become one of the most useful tools for studying this kind of problem in a simple way. Ever since the 1970s, the Brazilian disk has been commonly used for mixedmode fracture testing of a cracked component; ${ }^{23,24}$ 30 years later, Torabi started to use a modified version of the original Brazilian disk, the U-notched Brazilian disc specimen, to understand the triaxial stress state around U-notches under mixed-mode loading. ${ }^{15,25}$

The aim of this paper is to analyse when cracks can nucleate in a place near the notch tip in a zone where the stress is not singular. First of all, some experimental cases recently presented by Lorenzino et al. ${ }^{9}$ were analysed according to the implicit gradient (IG) theory. ${ }^{13,17,18}$ Furthermore, in this article, the authors investigate the geometries of different shaped $U$-notches and with a fillet radius. U-notches characterised by these differing geometries were subjected to mode I loading or predominant mode II loading. Focusing on the position of the maximum first principal stress, we investigated how the location of the maximum equivalent stress obtained with the implicit gradient approach changed as a function of the geometry parameters.

\section{THEORETICAL FRAMEWORK}

The authors present an approach capable of estimating the fatigue life of notched structures and welded joints based on an effective stress value computed numerically by solving the Helmholtz differential equation linked to the implicit gradient method. ${ }^{26}$ When it is not possible to evaluate the local maximum stress because the stress has become singular, it is convenient to define an effective local stress as the average of the stress inside a process zone. ${ }^{5,27}$ Nonetheless, the implicit gradient method supports the idea that the damage should be related to an average value on all the body; ${ }^{28}$ when calculating this average, the stress values near to the critical point have a greater impact than the distant stress field. With a non-local approach, we can evaluate the local effective stress $\sigma_{\text {eff }}$ and then compare it with the reference 


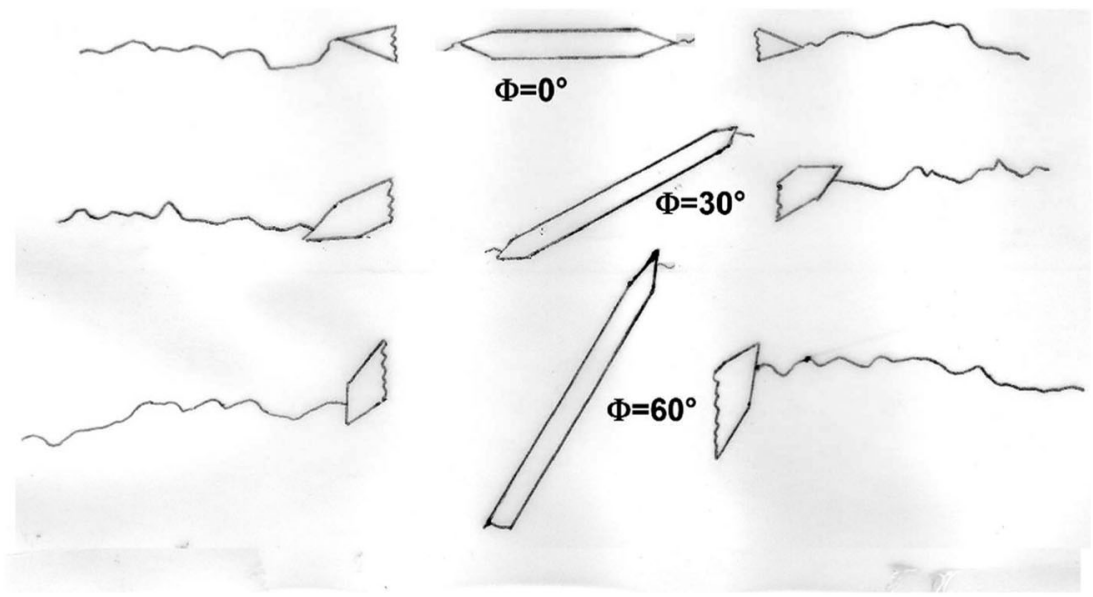

Fig. 3 Cracks nucleated near the notch tip observed at the fatigue limit in JIS-S15C by Lorenzino et al. ${ }^{9}$ (defect rotated at a tilt angle $\Phi$ and zoom at notch tip).

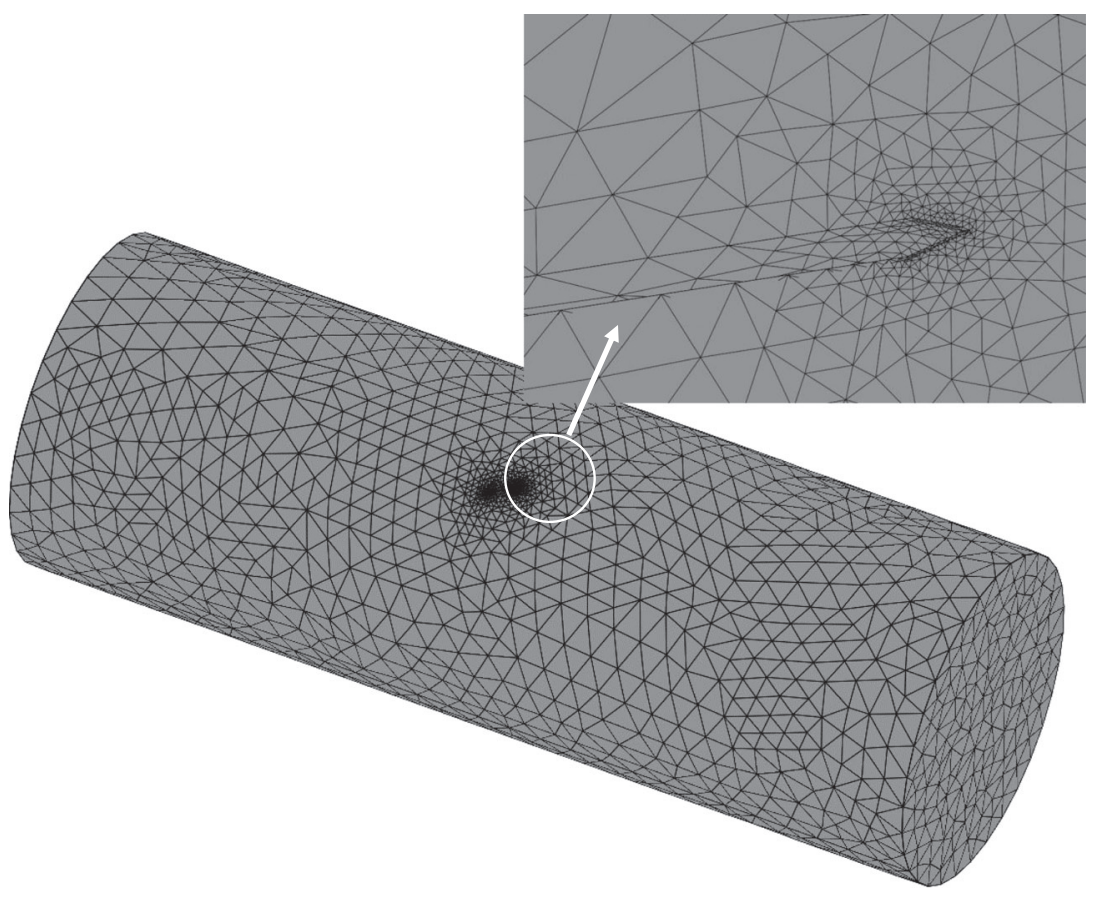

Fig. 4 Mesh used in FE analysis.

resistance of the base material. A non-local approach resolves the assessments of effective stress as:

$$
\sigma_{e f f, a v}(P)=\frac{\int_{V} \alpha(P, Q) \sigma_{e q}(Q) d V}{\int_{V} \alpha(P, Q) d V}
$$

where $\mathrm{Q}$ is a point inside volume $\mathrm{V}$ and the equivalent stress $\sigma_{\mathrm{eq}}$ is a function of the stress tensor (in our case is the first principal stress). The weight function $\alpha$ is an isotropic function of the distance $|P Q|$, which vanishes as distance $|P Q|$ increases. As an example, for a Gaussian weight function $\alpha=\frac{e^{\frac{\|\mathrm{PQ}\|^{2}}{2 L^{2}}}}{2 L^{2}}$ where $\mathrm{L}$ is a parameter relate to the material. ${ }^{29}$

To overcome the evaluation of integral (1) for each point, one can solve the associate Helmholtz differential equation obtained by simplifying the evaluations of average stress over the volume that includes a constant representing the characteristic length $c:{ }^{30}$ 


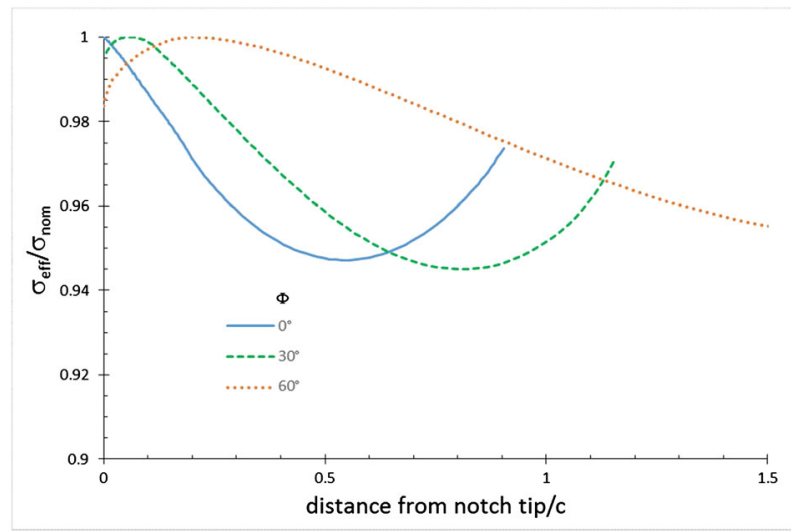

Fig. 5 Effective stress $\sigma_{\text {eff }}$ in dimensionless form along the crack border $(c=0.28 \mathrm{~mm})$.

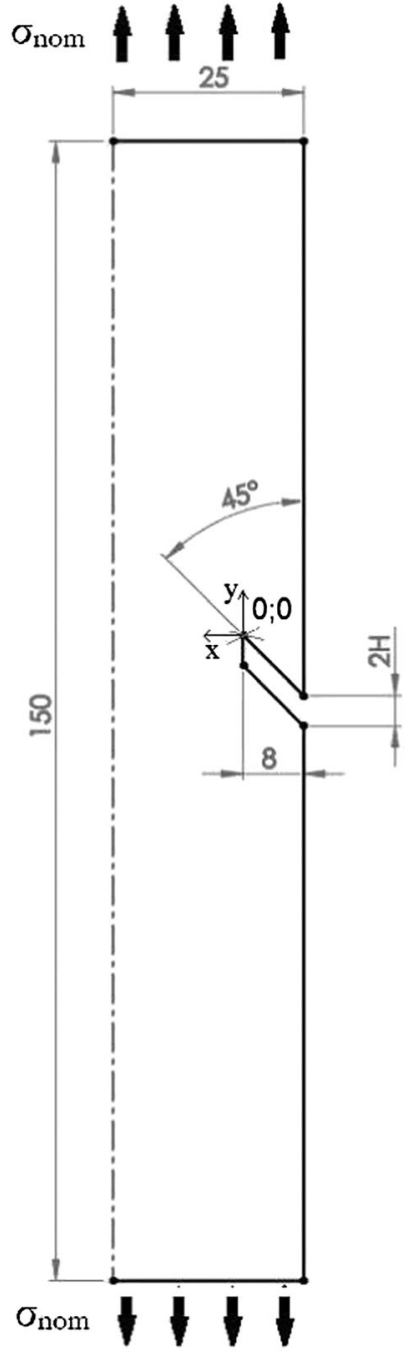

a)

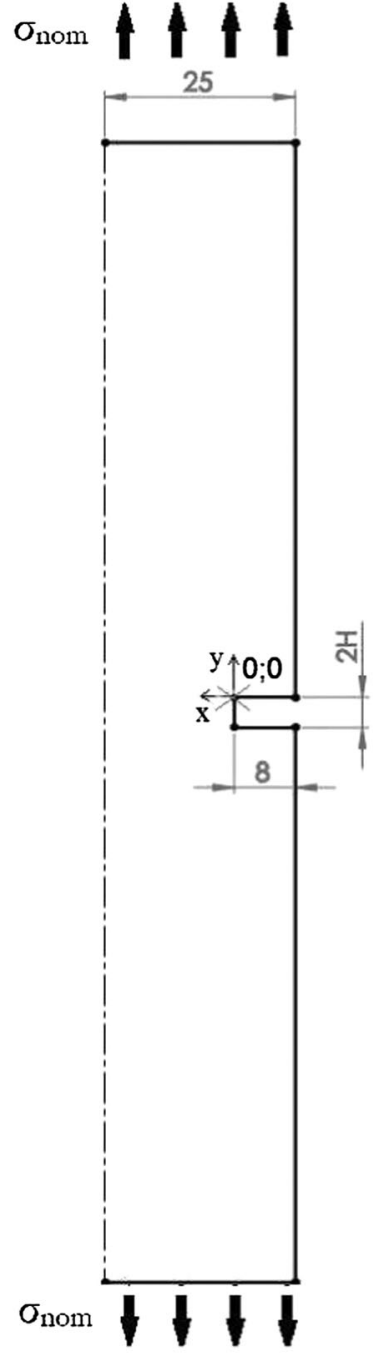

b)
Fig. 6 Sharp notch geometries (the dotted line is a symmetry axis, dimensions in $\mathrm{mm}$ ).

$$
\begin{gathered}
\sigma_{\text {eff }}=\mathrm{c}^{2} \nabla^{2} \sigma_{\text {eff }}=\sigma_{\text {eq }} \\
\nabla \sigma_{\text {eff }} \cdot \mathrm{n}=0
\end{gathered}
$$

The characteristic length $\mathrm{c}$ is an internal length scale and therefore the gradient formulation takes into account the scale effect (for welded structures made of steel $c$ was evaluated equal to $0.2 \mathrm{~mm}^{27}$ ). Eq. (1) can also be obtained by applying the theory of gradient elasticity as proposed in reference. ${ }^{31}$

As shown in equation (2), the only variable of this equation is the effective stress $\sigma_{\text {eff. }}$. This stress is related to the effective stress $\sigma_{\text {eff,av }}$ evaluated by means of Eq. (1). Equation (3) is the natural boundary condition. ${ }^{31}$ In Eq. (2) the equivalent stress can be evaluated by means of the liner elastic theory or by considering non-linear proprieties of material as recently proposed in reference. ${ }^{32}$ In this paper, the stress analysis takes into account a linear elastic material. It is important to consider that, if the first principal stress is taken as $\sigma_{\text {eq }}$, in general, the location of maximum value of $\sigma_{\text {eff }}$ not agree with that of the first principal stress. The numerical solution of Eq. (1) can be obtained by means of finite element (FE) software able to solve partial differential equation in a volume. However, at the notch tip an analytical or simplified numerical solution can be obtained. ${ }^{33}$

As an example, for a sharp $\mathrm{V}$-notch under mode I loading, Figs. 1 reports the value of $\sigma_{\text {eff }}$ against the distance from the notch tip. $\sigma_{\mathrm{eq}}$ was the principal stress and it became singular near the tip. On the contrary, $\sigma_{\text {eff }}$ is a continuous function also at the notch tip.

\section{NUCLEATION AROUND THE CRACK TIP}

In a recent paper, Lorenzino et al., ${ }^{9}$ proposed an accurate experimental study of small inclined defects with respect to the loading direction obtained in the surface of a cylindrical body under tensile loading. The researchers considered a tensile load around the fatigue limit of materials and obtained a non-propagating crack nucleated near the notch tip. Two types of carbon steels were used, JIS-S15C and JIS-S45C. The slit was tilted at a $\phi$ angle with respect to the perpendicular line to the cylinder axis. The $\phi$ angle was of 0,30 and 60 degrees, respectively. Fig. 2 shows the three types of defects analysed in this paper by means of the implicit gradient approach. The fracture surfaces of the specimens were observed using a scanning electron microscope. Fig. 3, shows the non-propagating cracks observed at the fatigue limit in the case of specimens made of JIS-S15C. For $\varphi=0^{\circ}$, the crack nucleated exactly at the notch tip. For $\varphi=30^{\circ}$, the crack slightly moved from the notch tip. In the case of $\varphi=60^{\circ}$ clearly appears that the crack nucleates in a different place than the notch tip. 


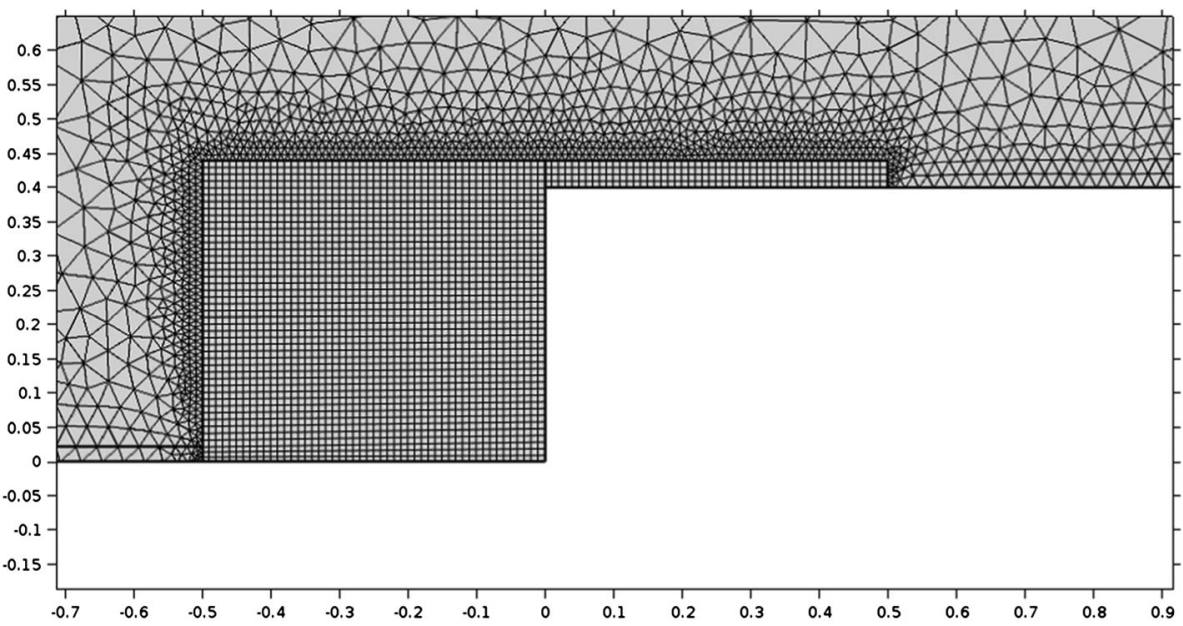

Fig. 7 Mesh detail: smallest element size $=0.01 \mathrm{~mm}, \mathrm{H}=0.4 \mathrm{~mm}$, axis scale in millimetres.

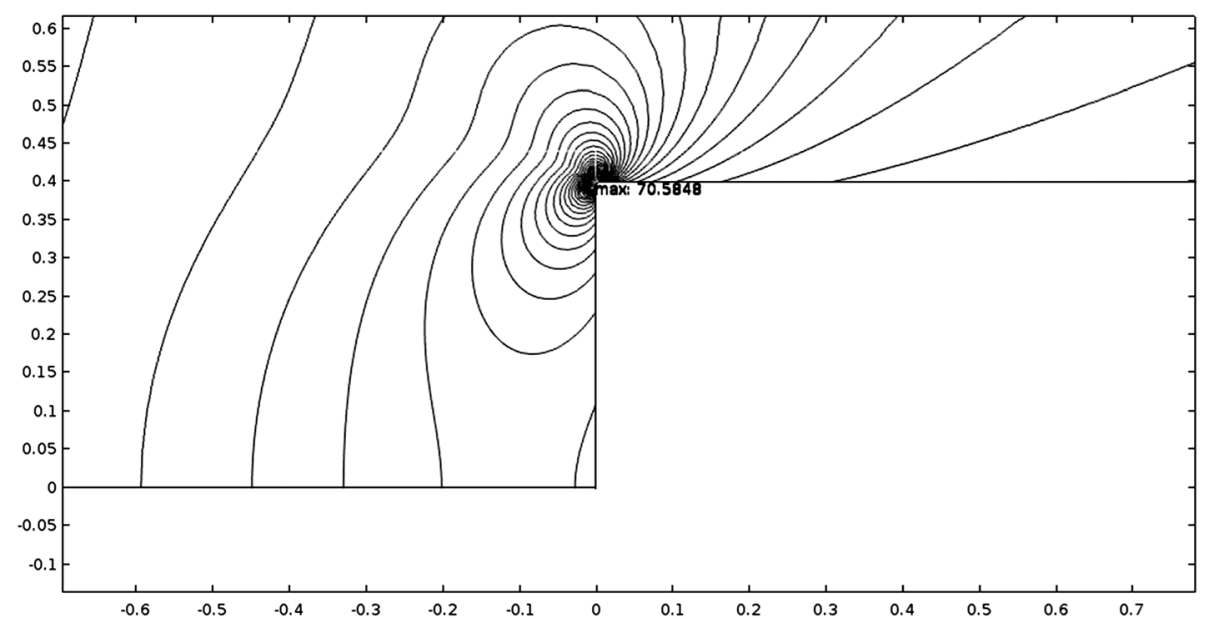

Fig. 8 Isolines graph of first principal stress $\sigma_{1} / \sigma_{\text {nom }}(H / c=2$, axis scale in millimetres).

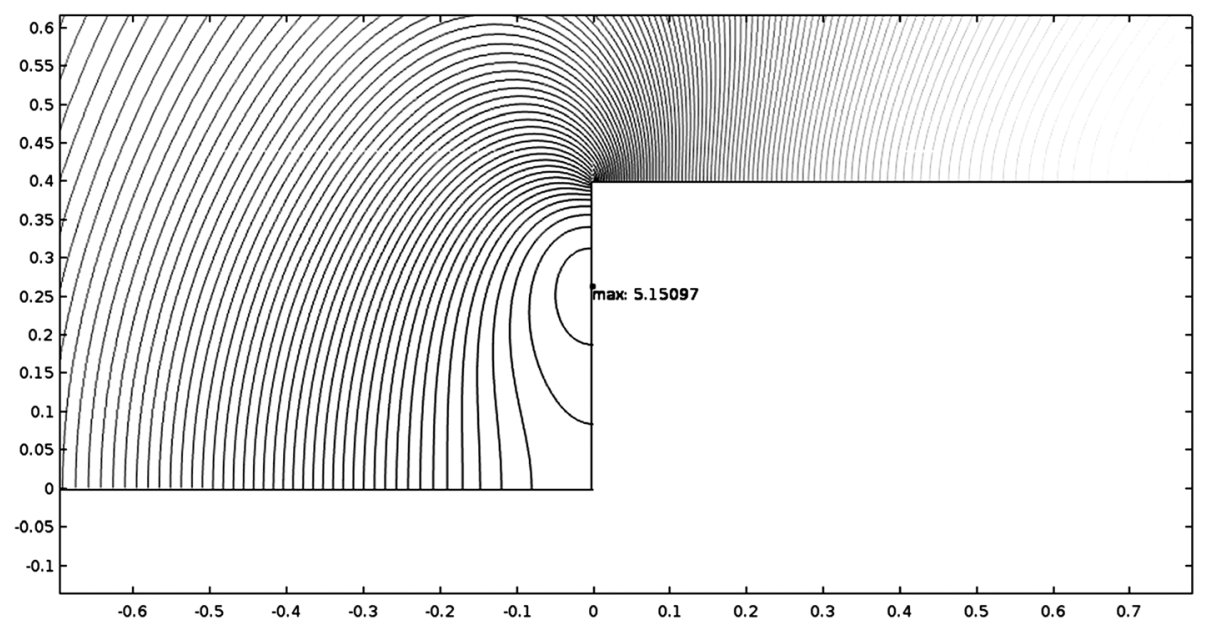

Fig. 9 Isolines of effective stress $\sigma_{\text {eff }} / \sigma_{\text {nom }}(\mathrm{H} / \mathrm{c}=2$, axis scale in millimetres). 

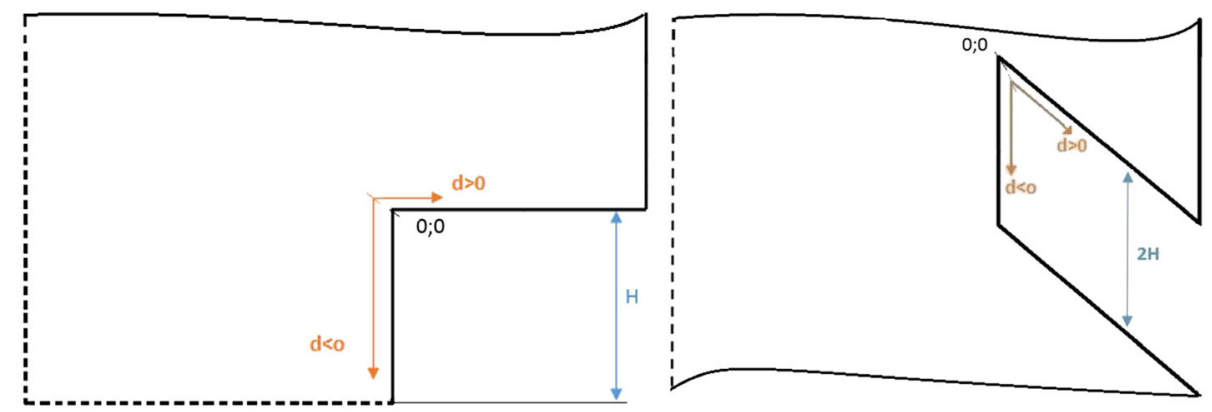

Fig. 10 Local coordinate systems for sharp notches: a) notch perpendicular to the load direction; b) inclined notch.

For the evaluation of characteristic length $c$ of JISS15C steel, unfortunately, there is not enough data in reference ${ }^{9}$ for a direct calculation. In this case, it is possible to estimate the El-Haddad $\mathrm{a}_{0}$ parameter ${ }^{34}$ as equal to the non-propagating crack value that was measured by Lorenzino et al. that results about $0.5 \mathrm{~mm}$. By recalling that there is a linear relationship between $c$ and $\mathrm{a}_{0}{ }^{18}$ :

$$
\mathrm{c}=\mathrm{z} \cdot \mathrm{a}_{0}
$$

the value of parameter $z$ is 0.55 if $\sigma_{\mathrm{eq}}$ is set equal to the maximum principal stress $\sigma_{1}$. So that a value of $c$ equal to $0.28 \mathrm{~mm}$ can be assumed for the JIS-S15C steel.

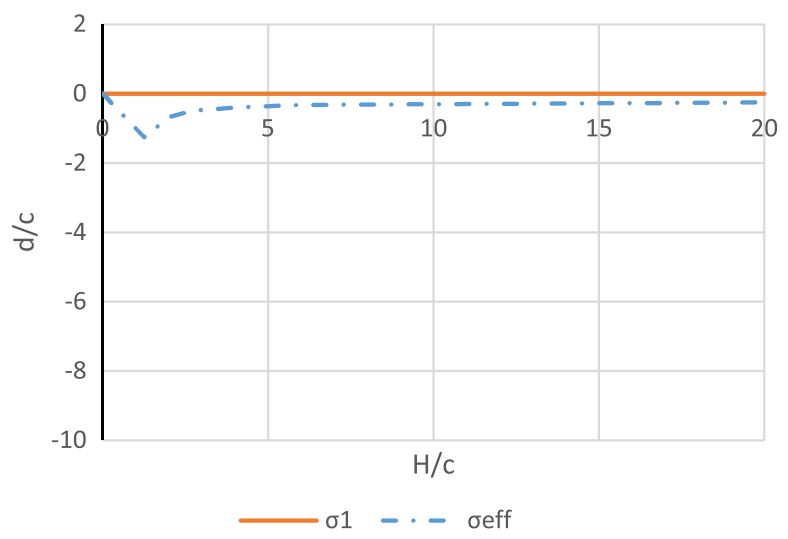

a): Notch perpendicular to the load direction

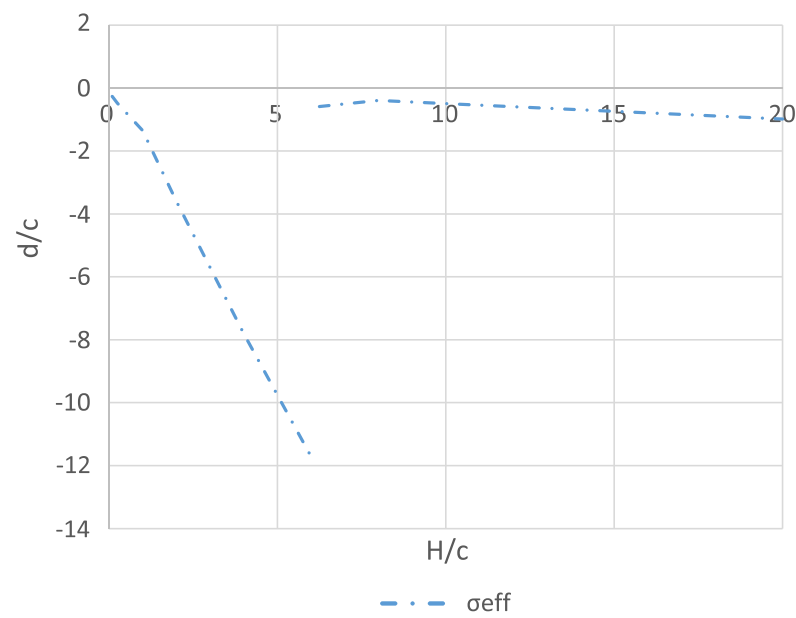

b): Inclined notch

Fig. 11 Position of maximum principal stress and the maximum effective stress for a sharp notch perpendicular to the load direction (a) and for an inclined sharp notch (b). 


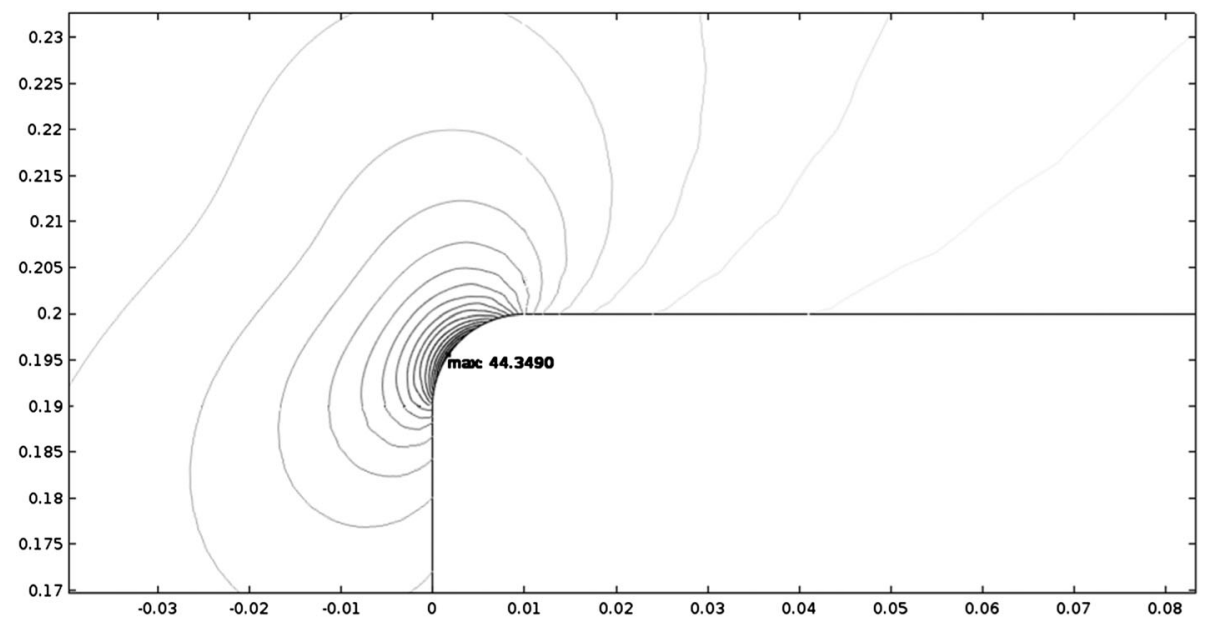

Fig. 12 Isolines of first principal stress in non-dimensional form $\sigma_{1} / \sigma_{\text {nom }}$ for a rounded notch $((\mathrm{H}-\mathrm{r}) / \mathrm{c}=0.95, \mathrm{r} / \mathrm{c}=0.05$, axis scale in millimetres $)$.

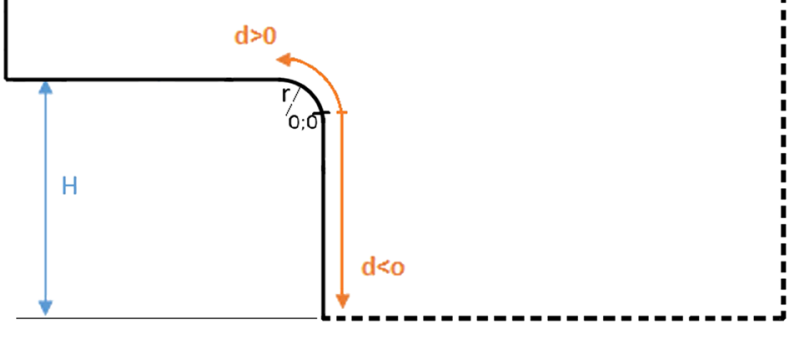

Fig. 13 Coordinate system used for the representation of maximum stress point location in rounded notches.

The crack was considered as elliptical and its exact shape at the notch tip was obtained from the photos reported in Ref.. ${ }^{9}$ As an example, Figs. 4 reports the mesh used in the tridimensional FE analysis. The mesh was refined only near the tip of the defect, usually the minimum size is $c / 2$. Fig. 5 shows the trend of the effective stress $\sigma_{\text {eff }}$ along the crack border on the external surface of the cylinder. The maximum of the effective stress $\sigma_{\text {eff }}$ is located at the notch tip only in the case of the $\phi$ angle equal to 0 degrees. In the other cases, the maximum of effective stress $\sigma_{\text {eff }}$ is located along the crack border as appears in the experimental cases of Figs. 3.

\section{RECTANGULAR NOTCHES}

In order to systematically analyse the effect of a shift of the maximum effective stress with respect to the notch tip in the following section we consider some geometrical cases of two-dimensional sharp rectangular notches. The characteristic length $c$ is taken equal to $0.2 \mathrm{~mm}$ for all parametric analysis

\section{Sharp notches}

Two macrosets of notch geometry were created with variable notch height $2 \cdot \mathrm{H}$ as reported in Figs. 6 . The first set is based on a horizontal notch with a fixed depth (Figs. 6a), and the second set is based on a notch of the same depth, but with the orientation of the notch set at 45 degrees (Figs. 6b). The half notch height $\mathrm{H}$ varies between 0 and $4 \mathrm{~mm}$ and a nominal uniform unitary tensile load is considered.

To make a proper model, we created a mesh with linear triangular elements and a mapped mesh zone over the notch tip. For the horizontal notch, the model was created with symmetry constraints in the mid-section of the notch. An example of the refinement of mesh used near the tip is reported in Figs. 7.

The first principal stress $\sigma_{1}$ in notched geometries is maximum at the notch tip as appears in Figs. 8.

On the contrary, the maximum value of $\sigma_{\text {eff }}$ is clearly located in the mid-section of the notch until $H / c=1.5$ (see Figs. 9). As can be seen in this example, the maximum value of $\sigma_{\text {eff }}$ is located in an unexpected position, and its location moves as $H$ changes (see Figs. 11).

We can easily create a coordinate system to locate the point of maximum stress and graph its position as reported in Figs. 10 (curvilinear coordinate dimension $d$ ).

With regards to the trend of the maximum value of $\sigma_{\text {eff }}$ as a function of $H / c$, Figs. 11 shows the shift of the maximum effective stress. In Figs. 11 it is clear that the 


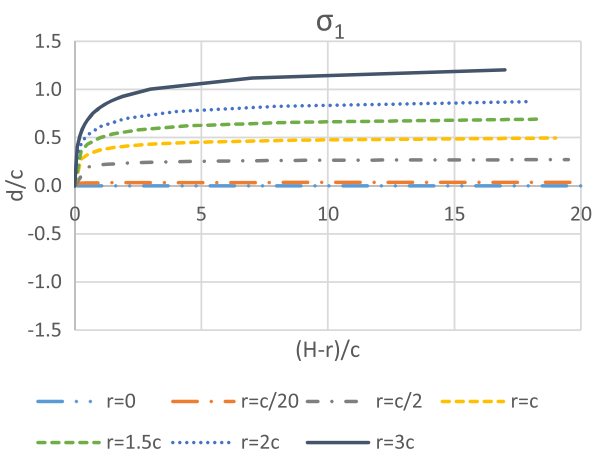

a)

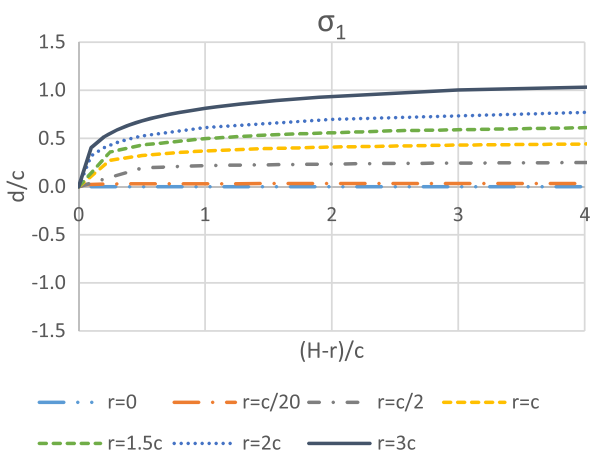

c)

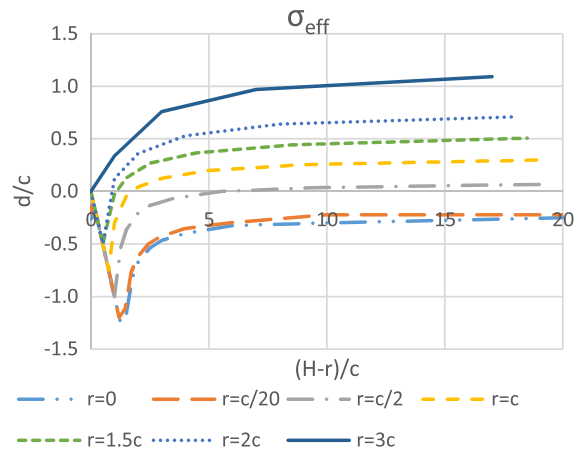

b)

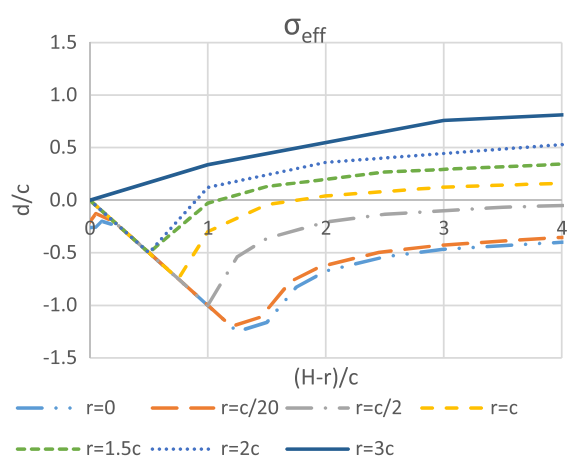

d)

Fig. 14 a-b) Position of the maximum principal stress and of the maximum effective stress; c-d) Zoom between 0 and 4.

Table 1 Position of the maximum (Max) effective stress $\sigma_{\text {eff }}$ related to H/c (sharp notches, $\mathrm{x}$ and $\mathrm{y}$ refered to Figs. $6, \mathrm{c}=0.2 \mathrm{~mm}$ )

\begin{tabular}{lrrrr}
\hline & \multicolumn{4}{c}{ Position of Max $\sigma_{\text {eff }}$} \\
$\mathrm{H} / \mathrm{c}$ & $\mathrm{x}[\mathrm{mm}]$ & $\mathrm{y}[\mathrm{mm}]$ & $\mathrm{x}[\mathrm{mm}]$ & $\mathrm{y}[\mathrm{mm}]$ \\
\hline 0 & -0.05 & 0.00 & -0.01 & 0.00 \\
0.1 & -0.02 & -0.02 & 0.01 & -0.05 \\
0.25 & 0.00 & -0.05 & 0.00 & -0.09 \\
0.5 & 0.00 & -0.10 & 0.00 & -0.15 \\
1 & 0.00 & -0.20 & 0.00 & -0.27 \\
2 & 0.00 & -0.14 & 0.00 & -0.72 \\
4 & 0.00 & -0.08 & 0.00 & -1.56 \\
6 & 0.00 & -0.06 & 0.00 & -2.34 \\
10 & 0.00 & -0.06 & 0.00 & -0.10 \\
20 & 0.00 & -0.05 & 0.00 & -0.20 \\
\hline
\end{tabular}

"hot spot" move from the location of singularity. However, the shift has the same order of the characteristic length $c$ for both perpendicular and inclined notch.

\section{Fillet notches}

As the notched geometries with a fillet radius are concerned, $\sigma_{1}$ does not reach its maximum in the point where the fillet starts, but it moves in the curvilinear part of the notch as shown in Figs. 12. As for the sharp notches, we
Table 2 Position of the maximum (Max) effective stress $\sigma_{\text {eff }}$ and $\sigma_{1}$ related to $(\mathrm{H}-\mathrm{r}) / \mathrm{c}$ for $\mathrm{r}=\mathrm{c} / 20$ (rounded notches, $\mathrm{x}$ and $\mathrm{y}$ refered to Figs. $6, \mathrm{c}=0.2 \mathrm{~mm}$ )

\begin{tabular}{llrrr}
\hline & \multicolumn{2}{c}{ Position of Max. $\sigma_{1}$} & \multicolumn{2}{c}{ Position of Max. $\sigma_{\text {eff }}$} \\
$(\mathrm{H}-\mathrm{r}) / \mathrm{c}$ & $\mathrm{x}[\mathrm{mm}]$ & $\mathrm{y}[\mathrm{mm}]$ & $\mathrm{x}[\mathrm{mm}]$ & $\mathrm{y}[\mathrm{mm}]$ \\
\hline 0 & 0.0000 & 0.0000 & -0.0416 & 0.0000 \\
0.05 & 0.0009 & 0.0040 & -0.0157 & -0.0100 \\
0.45 & 0.0015 & 0.0053 & 0.0000 & -0.0900 \\
0.95 & 0.0018 & 0.0057 & 0.0000 & -0.1900 \\
1.45 & 0.0019 & 0.0058 & 0.0000 & -0.2225 \\
1.95 & 0.0019 & 0.0058 & 0.0000 & -0.1263 \\
3.95 & 0.0021 & 0.0061 & 0.0000 & -0.0710 \\
5.95 & 0.0021 & 0.0061 & 0.0000 & -0.0595 \\
9.95 & 0.0022 & 0.0062 & 0.0000 & -0.0442 \\
19.95 & 0.0022 & 0.0062 & 0.0000 & -0.0443 \\
\hline
\end{tabular}

can easily create a coordinate system located at the tangent point to indicate the position of maximum stress in the parametric analysis (see Figs. 13).

Figs. 14 shows that also for fillet notches $\sigma_{\text {eff }}$ moves around the middle of the two tangent points.

It is extremely difficult to consider the fillet radius in inclined notches due to the different opening angles of the two notch tips: one tip has an opening angle of $135^{\circ}$ and can support almost any radius, whereas the other tip has a very sharp angle of $45^{\circ}$ 
and cannot support large radii for the lower values of $\mathrm{H}$. The results of $\mathrm{FE}$ analysis show that the maximum value of effective stress is always located in the sharpest angle, very close to the tip and independent of the notch radius.

Finally, in order to underline the shift of the maximum effective stress with respect to the first principal stress, Tables 1 and 2 propose a detailed comparison between the position of two stresses for sharp and rounded notches.

\section{CONCLUSIONS}

The main conclusions of the paper can be summarised as follows:

- With regards to small sharp defects like semi-elliptical cracks, the implicit gradient approach is able to capture the shift, from the notch tip, of the initiation crack point as experimentally observed when the local stress field is not symmetric;

- With regards to a plate weakened by a sharp rectangular notch under mixed loading (I plus II) the location of maximum effective stress moves from the place where the elastic stress field becomes singular. The shift is of the same order of the size of characteristic length $c$ for both mode I and dominant mode II loading.

- From the parametric analysis, it is possible to evaluate the width of the rectangular notch in order to have the fracture initiation point in the notch wall or at the notch tip. However, since the notch size and radius are very small, from an experimental point of view it may not be clear or easy to locate the effective starting point of the cracks.

\section{REFERENCES}

1 Westergaard, H. M. (1934) Stresses at a crack, size of the crack and the bending of reinforced concrete. Proc. American Concrete Institute, 30, 93-102.

2 Irwin, G. R. (1957) Analysis of Stresses and Strains Near the End of a Crack Traversing a Plate. 7. Appl. Mech., 24, 361-364.

3 Erdogan, F. and Sih, G. C. (1963) On the Crack Extension in Plates Under Plane Loading and Transverse Shear. 7. Basic Eng., ASME, 85, 519-527.

4 Sih, G. C. (1974) Strain-energy-density factor applied to mixedmode crack problem. Int. 7. Fract., 10, 305-321.

5 Neuber, H. (1957) Kerbspannungslehre, Springer: Berlin.

6 Pilkey, W. D. and Pilkey, D. F. (2008) Peterson's stress concentration, 3rd ed, John Wiley \& Sons, Inc., Hoboken, New Jersey.

7 Noda, N. A., Takase, Y. and Monda, K. (1997) Stress concentration factors for shoulder fillets in round and flat bars under various loads. Int. 7. Fatigue, 19, 75-84.

8 Waldman, W., Heller, M. and Chen, G. X. (2001) Optimal freeform shapes for shoulder fillets in flat plates under tension and bending. Int. 7. Fatigue, 23, 509-523.
9 Lorenzino, P., Okazaki, S., Matsunaga, H. and Murakami, Y. (2015) Effect of small defect orientation on fatigue limit of carbon steels. Fatigue Fract. Eng. Mater. Struct., 38, 1076-1086.

10 Radaj, D., Sonsino, C. M. and Fricke, W. (2006) Fatigue assessment of welded joints by local approaches, Second ed, Woodhead Publishing: Cambridge, UK.

11 Radaj, D. and Zhang, S. (1995) Fracture initiation outside the slit tip. Eng. Fract. Mech., 50, 309-310.

12 Yuuki R., Ohira T. (1989) Development of the method to evaluate the fatigue life of spot-welded structures by fracture mechanics, IIW Doc III-928-89/XIII-1358-89

13 Tovo, R. and Livieri, P. (2011) A numerical approach to fatigue assessment of spot weld joints. Fatigue Fract. Eng. Mater. Struct., 34, $32-45$.

14 Williams, M. L. (1952) Stress singularities resulting from various boundary conditions in angular corners of plates in extension. 7. Appl. Mech., 19, 526-528.

15 Torabi, A. R. and Berto, F. (2014) Strain energy density to assess mode II fracture in U-notched disk-type graphite plates. Int. F. Damage Mech., 23, 917-930.

16 Berto, F. and Lazzarin, P. (2009) A review of the volume-based strain energy density approach applied to $\mathrm{V}$-notches and welded structures. Theor. Appl. Fract. Mech., 52, 183-194.

17 Maggiolini, E., Livieri, P. and Tovo, R. (2015) Implicit gradient and integral average effective stresses: Relationships and numerical approximations. Fatigue Fract. Eng. Mater. Struct., 38, 190-199.

18 Tovo, R., Livieri, P. and Benvenuti, E. (2006) An implicit gradient type of static failure criterion for mixed-mode loading. Int. 7 . Fract., 141, 497-511.

19 Taylor, D. (1999) Geometrical effects in fatigue: a unifying theoretical model. Int. F. Fatigue, 21, 413-420.

20 Susmel, L. and Taylor, D. (2007) A novel formulation of the theory of critical distances to estimate lifetime of notched components in the medium-cycle fatigue regime. Fatigue Fract. Eng. Mater. Struct., 2007(30), 567-581.

21 Zappalorto, M. and Lazzarin, P. (2011) Stress fields due to inclined notches and shoulder fillets in shafts under torsion. 7 . Strain Anal. Eng., 46, 187-199.

22 Majima, T., Tobita, T. and Kimizuka, Y. (1987) Experimental Investigation on Interference Effect of Notch on Strength of Notched Bar with Double U-Notches of Unequal Depth and Radius. 7. Soc. Mater. Sci., 36, 871-877.

23 Awaji, H. and Sato, S. (1978) Combined mode fracture toughness measurement by the disc test. F. Eng. Mater. Tech., 100, $175-182$.

24 Awaji, H. and Kato, T. (1998) Griffith criterion for mode II fracture of ceramics. In: Experimental Mechanics (Edited by I. M. Alison), Balkema: Rotterdam, pp. 1199-1204.

25 Torabi, A. R., Fakoor, M. and Pirhadi, E. (2014) Fracture analysis of U-notched disc-type graphite specimens under mixed mode loading. Int. 7. Solids Struct., 51, 1287-1298.

26 Tovo, R. and Livieri, P. (2007) An implicit gradient application to fatigue of sharp notches and weldments. Eng. Fract. Mech., 74, $515-526$.

27 Livieri, P. and Tovo, R. (2004) Fatigue limit evaluation of notches, small cracks and defects: an engineering approach. $\mathrm{Fa}$ tigue Fract. Eng. Mater. Struct., 27, 1037-1049.

28 Pijaudier-Cabot, G. and Bažant, Z. P. (1987) Nonlocal Damage Theory. F. Eng. Mech-Asce, 10, 1512-1533.

29 Peerlings, R. H. J., Geers, M. G. D., de Borst, R. and Brekelmans, W. A. M. (2001) A critical comparison of nonlocal and gradient-enhanced softening continua. Int. F. Solids Struct., 38, 7723-7746. 
30 Peerlings, R. H. J., de Borst, R., Brekelmans, W. A. M. and de Vree, J. H. P. (1996) Gradient enhanced damage for quasibrittle material. Int. 7. Numer. Methods Eng., 39, 3391-3403.

31 Miihlhaus, H.-B. and Aifantis, E. C. (1991) A variational principle for gradient plasticity. Int. 7. Solids Struct., 28, 845-857.

32 Livieri, P., Salvati, E. and Tovo, R. (2016) A non-linear model for the fatigue assessment of notched components under fatigue loadings. Int. 7. Fatigue, 82, 624-633.
33 Tovo, R. and Livieri, P. (2008) An implicit gradient application to fatigue of complex structures. Eng. Fract. Mech., 75, 1804-1814.

34 El Haddad, M. H., Topper, T. H. and Smith, K. N. (1979) Fatigue crack propagation of short cracks. F. Eng. Mater-T $A S M E$, 101, 42-45. 